\title{
Internalized weight bias and cortisol reactivity to social stress
}

\author{
F. U. Jung ${ }^{1}$ (D) $\cdot$ Y. J. Bae ${ }^{2}$ • J. Kratzsch ${ }^{2}$ · S. G. Riedel-Heller ${ }^{1}$ • C. Luck-Sikorski ${ }^{3,4}$
}

Published online: 25 October 2019

(C) The Psychonomic Society, Inc. 2019

\begin{abstract}
Weight-associated stigmatization and discrimination may induce chronic stress in individuals with obesity. As a consequence, this stressor may cause an imbalance of HPA stress axis leading to increased eating behavior, and ultimately, weight gain. However, the direct link between internalized weight bias and stress response to acute stressors via cortisol secretion has not been investigated so far. Therefore, the purpose of this study was to investigate the interaction between internalized weight stigma as a stressor and cortisol reactivity in an acute psychosocial stress situation induced by the Trier Socials Stress Test for groups (TSST-G). Participants with BMI $>30 \mathrm{~kg} / \mathrm{m}^{2}(\mathrm{n}=79)$ were included in the study. Results reveal that while individuals with low internalized stigma reacted as predicted with an increase in cortisol secretion to acute psychosocial stress, individuals with medium or high internalized stigma did not show a typical cortisol response. However, these findings depend on the several factors, for instance on gender. In sum, acute stress in individuals with internalized weight bias seems to blunt HPA axis reactions to acute psychosocial stress. The study contributes to the understanding of the psychological and endocrinological consequences of internalized weight bias and underlines the importance of interventions to reduce stigmatization.
\end{abstract}

Keywords Cortisol $\cdot$ Stigma $\cdot$ Obesity $\cdot$ Trier socials stress test $\cdot$ Stress

\section{Introduction}

The recent increase in the prevalence of obesity and overweight has been observed in many different countries worldwide (Abarca-Gómez, Abdeen, Hamid, Abu-Rmeileh, Acosta-Cazares, Acuin, 2017). In this context, psychosocial stress has been suggested to be an important risk factor for development of overweight and obesity by acting as a chronical stressor (Tomiyama, 2014). Usually, stress can be seen as a typical response to a variety of strains or stimuli. The human body reacts to stress by stimulating the hypothalamic-

F. U. Jung

franziska.jung@medizin.uni-leipzig.de

1 Institute of Social Medicine, Occupational Health and Public Health (ISAP), Medical Faculty, University of Leipzig,

Philipp-Rosenthal-Str. 55, 04103 Leipzig, Germany

2 Institute of Laboratory Medicine, Clinical Chemistry and Molecular Diagnostics, University of Leipzig, Paul-List Strasse 13-15, D-04103 Leipzig, Germany

3 Integrated Research and Treatment Center (IFB) Adiposity Diseases, University Hospital Leipzig, Philipp-Rosenthal-Str. 27, 04103 Leipzig, Germany

4 SRH University of Applied Health Sciences, Neue Straße 28-30, 07548 Gera, Germany pituitary (HPA) axis leading to cortisol secretion from the adrenal cortex. This reaction should diminish after successfully coping with the stressful event (Papadimitriou \& Priftis, 2009). However, studies suggest that individuals with obesity may respond differently (for a review: van der Valk, Savas, \& van Rossum, 2018), depending on individuals characteristics, such as mental distress or genetics. Whether stress response can be seen as a cause or a consequence with regard to obesity or simply weight gain is still not fully understood and may depend on several physiological aspects, such as chronic lowgrade inflammation or levels of leptin (Foss \& Dyrstad, 2011). Research has shown that cortisol activity differs between individuals with obesity and normal-weight controls, suggesting a dysregulated activity of the HPA axis in individuals with obesity (Herhaus \& Petrowski, 2018). In other words, altered cortisol activity may be a consequence of obesity. On the other hand, there is evidence that obesity may be caused by stressinduced elevations in cortisol, which are associated with gaining weight, either by an overall increase in food intake or by increasing the preference for specific food types high in fat or sugar content in response to a stressful episode (Fardet \& Fève, 2014). Moreover, elevated glucocorticoid levels (such as cortisol levels) may contribute to the regulation of adipose tissue metabolism leading to visceral adiposity and increased risk for developing type 2 diabetes mellitus (Peckett, Wright, \& Riddell, 2011). The link between obesity and stress has 
been investigated by focusing on causation of stressful events. Chronic stress experienced by individuals with obesity may result from several psychosocial aspects. According to the social self-preservation model by Dickerson and Kemeny's (2004), we face many different type of stressors during our lives; however, those that are characterized by negative social evaluation can have the greatest impact on the HPA axis in terms of cortisol activity (Dickerson \& Kemeny, 2004; Woody, Hooker, Zoccola, \& Dickerson, 2018). In this context, social evaluative threats, such as stigmatization and discrimination, have been defined as negative attitudes and prejudice toward individuals with obesity and overweight that may be experienced verbally or nonverbally and occur in many different everyday life situations (Puhl \& Heuer, 2009). Sometimes, stigma and weight bias can be internalized by those affected, having negative influences on overall wellbeing and mental health (Pearl \& Puhl, 2018), which has been defined as internalized weight bias. The negative consequences of weight discrimination and self-stigmatization on physiological and psychological health have been recently reviewed in greater detail (Wu \& Berry, 2018). According to the Cyclic Obesity/Weight-Based Stigma-Model (Tomiyama, 2014), weight stigmatization can act as a chronic stressor leading to perpetuation of obesity and associated comorbidities, because weight bias and perceptions of weight stigma can abnormally alter cortisol levels (Himmelstein, Belsky, Angela, \& Tomiyama, 2015). A very recent study has demonstrated that individuals with obesity who have experienced being discriminated due to their body weight are generally more likely to have elevated cortisol levels compared with individuals who have not been stigmatized (Jackson, Kirschbaum, \& Steptoe, 2016). Another study that also investigated the association between weight stigma and cortisol did not find significant results, when variables, such as age or perceived stress, are controlled for (Wu, Berry, \& Schwartz, 2019). Research focusing on oxidative stress levels has shown that perception of weight stigma is positively correlated with morning serum cortisol and F2-isoprostane levels, which can in turn cause long-term damage by interrupting the activity of antioxidant enzymes (Tomiyama, Epel, McClatchey, Poelke, Kemeny, McCoy, Daubenmier, 2014). In a different study, which compared effects of video material with stigmatizing contents (Schvey, Puhl, \& Brownell, 2014), weightstigmatizing stimuli resulted in greater cortisol reactivity among female participants by affecting not only participants with overweight, but also normal-weight participants. Interestingly, human stress research suggests that individuals may be characterized as being low cortisol responders or high cortisol responders (Kirschbaum, Prüssner, Stone, Federenko, Gaab, Lintz, 1995; Schommer, Hellhammer, \& Kirschbaum, 2003). For example, one study (McCleary-Gaddy, Miller, Grover, Hodge, \& Major, 2019) showed that when placing individuals with overweight or obesity in a weight- stigmatizing employment setting, HPA activity was characterized by blunted cortisol responses, whereas normal-weight participants showed rather typical rising cortisol levels. Mechanisms are unclear but may be found in the degree of internalization. Self-stigmatization could result in a state of physiological arousal that in turn gives rise to metabolic abnormalities via cortisol secretion or alter biological pathways (Pearl, Wadden, Hopkins, Shaw, Hayes, Bakizada, 2017). According to McEwan (1998) to maintain physiological homeostasis, the body tries to adapt to potentially stressful challenges via "allostasis," making it possible to cope with a variety of stressors. However, this system may sometimes be overstimulated or not perform normally (i.e., allostatic load). This can cause disease, for instance due to frequent activation ("repeated hits"), lack of adaptation, prolonged stress response with no recovery, or inadequate stress responses (i.e., blunted cortisol response) (McEwen, 2006). In this context, allostatic load can be seen as a biological pathway that translates chronic stress into many different health outcomes often linked to obesity and overweight, affecting cardiovascular functioning, metabolism, immune system, as well as central nervous system (CNS) functioning (McEwen, 2006).

Therefore, the purpose of the current study was to further investigate how weight stigma negatively affects individuals with obesity and overweight by testing whether there is a direct link between internalized weight bias (IWB) and stress response via cortisol secretion. In light of the aforementioned literature, we expect that cortisol secretion is maladaptive in individuals with high internalized weight bias. Thus, we hypothesize that (1) individuals with high levels of weight bias internalization have higher cortisol levels at baseline but (2) show overall blunted cortisol levels in response to an acute psychosocial stressor.

\section{Methods}

\section{Participants}

Exclusion criteria are summarized in Table 1. Inclusion criterion for participants was having a body mass index (BMI) greater than $30 \mathrm{~kg} / \mathrm{m}^{2}$. BMI was assessed twiceduring telephone pre-screening and as part of the questionnaire-by asking for weight and height. Eightyseven participants were included in the study; however, of this total sample, we had to exclude three participants from final analysis due to missing data, three participants because they did not fulfill the requirement of an BMI $>30 \mathrm{~kg} / \mathrm{m}^{2}$, and two participants because they stated after the experiment that they have had weight loss surgery during the past, which could have influenced the stress response. Therefore, data from only 79 participants were included in the final analysis. 
Table 1 Exclusion criteria

$\begin{array}{ll}\text { - Substance or alcohol abuse (past } 2 \text { months) } & \text { - Neurological disease } \\ \text { - Pregnancy } & \text { - Hormonal dysregulation } \\ \text { - Taking hormonal contraceptives } & \text { - Virus infections } \\ \text { - More than } 5 \text { cigarettes/day } & \text { - Coronary disease, high } \\ & \text { blood pressure } \\ \text { - Paradontisis } & \text { - Diabetes } \\ \text { - Disease of bones, skeleton } & \text { - Coagulum } \\ \text { - Exogene glucocorticoid therapy } & \text { - Liver or kidney disease }\end{array}$

\section{Recruitment}

Participants were recruited on social media platforms and announcements in public places such as supermarkets. They were informed that the study is designed to investigate the perception of and coping with stress in general. They received an incentive (50 Euros) after their participation. If they agreed on taking part in the study, they were then contacted per mail or telephone with further information and appointments. In particular, they were instructed to refrain from doing any kind of sport 24 hours before the appointment. Additionally, they were instructed not to eat (especially no liquorice), smoke or drink either tea, coffee, or alcohol 2 hours before their appointment, because this can affect the results of the salivary sample (Hansen, Garde, \& Persson, 2008; Nicolson, 2008). The study was approved by the Ethical Committee of the Medical Faculty at the University of Leipzig (reference: 137-1625042016).

\section{Procedure}

The study took place at $3 \mathrm{pm}$ (start of the appointment, T0) and included the Trier Social Stress Test for Groups (TSST-G) (Allen, Kennedy, Dockray, Cryan, Dinan, Clarke, 2017; Dawans, Kirschbaum, \& Heinrichs, 2011), as well as questionnaires, which are described in greater detail below. The TSST-G is an experimental design that follows a standardized procedure during which biomarker samples, such as salivary cortisol can be taken at different time points. According to the literature, the TSST for groups is equivalent to the TSST for individuals, but rather cost-saving (Dawans et al., 2011). It allows for an experimental design that evokes acute psychosocial stress, which leads to a short-term increase in cortisol as a result of the perceived social-evaluative threat. In healthy subjects, this increase normalizes after 60 to 90 minutes (Kirschbaum, Pirke, \& Hellhammer, 1993). The TSST-G often is used as a reliable psychological protocol in stress research, because it follows a highly standardized protocol. Due to difficulties with scheduling appointments, participants were tested either in pairs $(\mathrm{N}=35)$ or groups of three $(\mathrm{N}=44)$. All participants in this study were naïve to the following procedure. Group size was therefore added as a covariate. Eating and brushing teeth were restricted for $30 \mathrm{~min}$ before sampling. The first saliva sample (baseline sample, T1) was collected after participant's arrival. At this stage, participants did not know about the tasks they had to face during TSST-G. Each testing group consisted of female or male participants only. The TSST-G itself consisted of three standardized stages: 1) welcoming and instructions by an experimenter, followed by 10 minutes of preparation for a mock job interview, 2) free speech (three minutes per participant), and 3) mathematical skill testing ( $3 \times 30$ seconds per participant). During the mock job interview, participants were told that the interview was videotaped. In addition, they had to perform their tasks in front of a committee, which consisted of a woman and a man wearing lab coats. During the interview, both committee members did not engage socially with the participants or gave any positive feedback. Moreover, participants were separated from each other by standing walls and they were instructed not to interact at any time. Participants were then escorted to another room where the second salivary cortisol sample (T2) was taken, and they were asked to fill out a questionnaire (resting phase). After that, the third and last salivary cortisol sample was taken (T3; Fig. 1). At the end of the resting phase (at T4), participants were debriefed and informed that neither the dictation device nor the video camera were recording at any time of the experiment.

The structure of the experimental design, as well as the timings of the individual phases of the experiment can be found in Fig. 1.

\section{Questionnaires}

The questionnaires which participants were asked to fill out during the resting phase included sociodemographic information, such as age, weight, height, and education. Weight and height were assessed using self-reports. To ensure eligibility, a question on previous diagnosis of eating disorders was added, asking: "Have you ever been diagnosed with one of the following disorders: Binge eating, Night eating or any other eating disorder? (yes/no)."

\section{Depressive symptoms}

In addition to that, depressive symptoms were surveyed using the German version of the Patient Health Questionnaire (PHQ-9) (Spitzer, Kroenke, \& Williams, 1999). This questionnaire consists of nine questions to be answered on a 4-point scale $(0=$ not at all; $3=$ nearly every day). Participants had to evaluate the following question for each complaint: "Over the last two weeks, how often have you been bothered by any of the following problems?" An exemplary complaint is: "Little interest or pleasure in doing things?" The German version was 


\begin{tabular}{|l|l|l|l|}
\hline \multicolumn{2}{|c|}{ Preparation } & \multicolumn{1}{c|}{ Test-Phase } & \multicolumn{1}{c|}{ Resting-Phase } \\
\hline $\begin{array}{l}\text { Welcoming, informed } \\
\text { consent, description of } \\
\text { the procedure }\end{array}$ & $\begin{array}{l}\text { Preparation of the } \\
\text { speech (10 min.) }\end{array}$ & $\begin{array}{l}\text { Trier Social Stress Test } \\
\text { (speech: 3 minutes/ppt; } \\
\text { mathematical task 3x30 } \\
\text { seconds/ppt) }\end{array}$ & $\begin{array}{l}\text { Recovery, } \\
\text { questionnaire, } \\
\text { debriefing }\end{array}$ \\
\hline
\end{tabular}

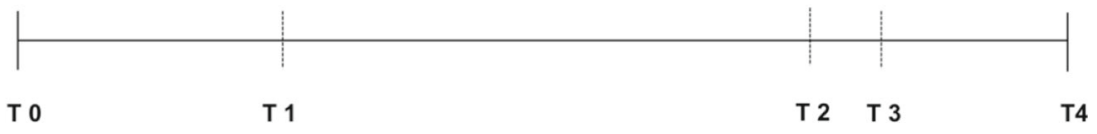

Note: $p p t=$ per participant, $\min =$ minute

Fig. 1 Time frame of the experimental design. Note: $p p t=$ per participant, $\min =$ minute

translated and validated by (Löwe et al., 2002), with good reliability (Cronbach $\alpha=0.88$ ).

\section{Weight bias internalization}

Internalized weight stigmatization was assessed using the Modified Weight Bias Internalization Scale (WBIS-M) (Durso, Latner, \& Hayashi, 2012). Participants are asked to respond to 11 items using a Likert-scale ( $1=$ strongly disagree, $7=$ strongly agree) to measure how much they agree with negative stereotypes and apply them to themselves by making negative judgements about their body weight. The WBIS-M scale has been translated into German, showing good psychometric properties (Hilbert, Baldofski, Zenger, Löwe, Kersting, Braehler, 2014). As has been suggested by Hilbert et al. (2014), item 1 should be removed from the analysis, which also improved reliability (Cronbach's $\alpha=0.91$ ). A mean score is calculated from the remaining ten items, which is then divided by the number of items. The greater this score, the higher the internalization of weight bias. Details on how to calculate sufficient cutoff points have been provided by a recent study (Puhl, Himmelstein, \& Quinn, 2018). The authors suggest constructing cutoffs for internalization based on (1) scoring one standard deviation (SD) below the mean of internalization or lower, (2) at the mean on internalization, and (3) one SD above the mean on internalization or higher. In this sample, the mean WBI score was 2.69 and the standard deviation was 1.19 (range: 0.2-5.2). Corresponding cutoffs were calculated and can be found in Table 2 .

\section{Chronic stress}

Chronic stress was assessed using the Screening Scale of the Trier Inventory for the Assessment of Chronic Stress (TICSSSCS,(Schulz \& Schlotz, 1999)) (Cronbach $\alpha=0.91$ ). This scale consists of 12 items that reflect experiences of stressful situations during everyday life. The prevalence of distress is assessed using a five-point scale $(0=$ never, $4=$ very often $)$ and the level of distress is then calculated using a sum-score.

\section{Salivary cortisol analysis}

Saliva samples were collected using Salivette ${ }^{\circledR}$ tubes for cortisol (Sarstedt, Sevelen, Switzerland). Instruction for saliva samples collection has been described in "Salivette ${ }^{\circledR}$ for Cortisol" (Sarstedt, Nümbrecht, Germany). All cortisol samples were transported to the laboratory in cooling boxes after collection. Collected saliva was centrifuged and aliquoted for measuring cortisol concentrations. Samples were stored at $-80{ }^{\circ} \mathrm{C}$ until ready for experimentation. Salivary cortisol was analyzed by LC-MS/MS as previously described (Bae,

Table 2 Sociodemographic characteristics of the sample measurements of depressive symptoms and chronic stress

\begin{tabular}{|c|c|}
\hline & Sample $(\mathrm{n}=79)$ \\
\hline Age & $\begin{array}{l}\text { Range: } 19-62 \text { years } \\
\text { M: } 32.4 \text { years (SD: } 10.2)\end{array}$ \\
\hline \multicolumn{2}{|l|}{ Gender, \%(n) } \\
\hline $\begin{array}{l}\text { male } \\
\text { female }\end{array}$ & $\begin{array}{l}31.6 \%(25) \\
68.4 \%(54)\end{array}$ \\
\hline Body mass index (BMI) & $\begin{array}{l}\text { Range: } 30.3-47.3 \\
\text { M: } 35.5 \text { (SD: } 4.2)\end{array}$ \\
\hline \multicolumn{2}{|l|}{ Education, \%(n) } \\
\hline $\begin{array}{l}<12 \text { years } \\
>12 \text { years } \\
\text { Not specified }\end{array}$ & $\begin{array}{l}35.4 \%(28) \\
53.2 \%(42) \\
11.4 \%(9)\end{array}$ \\
\hline \multicolumn{2}{|l|}{ Eating disorder, $\%(\mathrm{n})$} \\
\hline $\begin{array}{l}\text { Yes } \\
\text { No } \\
\text { Not specified }\end{array}$ & $\begin{array}{l}5.1 \%(4) \\
92.4 \%(73) \\
2.5 \%(2)\end{array}$ \\
\hline Symptoms of Depression (PHQ-9) & $\begin{array}{l}\text { Range: } 0-21 \\
\text { M: } 6.9 \text { (SD: 4.6) }\end{array}$ \\
\hline Weight bias internalization (WBI), \%(n) & Range: $0.2-5.2$ \\
\hline $\begin{array}{l}\text { Low }(\leq 1.8) \\
\text { Medium } \\
\text { High }(\geq 3.66)\end{array}$ & $\begin{array}{l}17.7 \%(14) \\
60.8 \%(48) \\
21.5 \%(17)\end{array}$ \\
\hline Chronic stress (TICS-SSCS) & $\begin{array}{l}\text { Range: } 14-52 \\
\text { M: } 32 \text { (SD: 10.2) }\end{array}$ \\
\hline
\end{tabular}


Gaudl, Jaeger, Stadelmann, Hiemisch, Kiess, 2016; Gaudl, Kratzsch, Bae, Kiess, Thiery, Ceglarek, 2016).

To investigate and determine the level of stress experienced during TSST-G, participants were categorized into nonresponders and responders based on the criterion of a cortisol increase of at least $1.5 \mathrm{nmol} / \mathrm{L}$ (Miller, Plessow, Kirschbaum, \& Stalder, 2013). Cortisol increase is hereby defined as the difference between peak cortisol and baseline cortisol level. If this difference is below $1.5 \mathrm{nmol} / \mathrm{L}$, participants can be categorized as nonresponders, if the cutoff is exceeded, participants can be categorized as responders.

\section{Statistical analysis}

The data were analyzed using Stata Version 13 SE and SPSS Version 23. Due to spontaneous cancellation of appointments made by participants, the TSST-G was conducted in groups of either two or three participants. Therefore, the variable "group size" was included as a control variable. To achieve normality, all cortisol data were normalized using log-transformation before further analysis was conducted.

Cortisol reactivity and recovery were determined by calculating two indices. These indices with regard to the area under the curve (AUC) were derived using formulas given by Pruessner et al. (2003): area under curve with regard to baseline $\left(\mathrm{AUC}_{\mathrm{G}}\right.$, total reactivity or output of cortisol) and area under curve with respect to increase $\left(\mathrm{AUC}_{\mathrm{I}}\right.$, change in total reactivity of cortisol due to TSST, i.e., sensitivity of the hormonal system). The formulas allow for individual adjustment due to different times between measurements (Fig. 2). This procedure ensures that differences based on sampling time (Fig. 1) are controlled for.

Differences between groups with regard to weight bias internalization and sociodemographic characteristics and influencing variables were examined using independent $t$ tests and chi-square tests. We conducted a linear regression in order to investigate whether chronic stress measured by

$$
\begin{gathered}
A U C_{G}=\sum_{i=1}^{n-1} \frac{\left(m_{(i+1)}+m_{i}\right) \times t_{i}}{2} \\
A U C_{I}=\left(\sum_{i=1}^{n-1} \frac{\left(m_{(i+1)}+m_{i}\right) \times t_{i}}{2}\right)-\left(m_{1} \times \sum_{i=1}^{n-1} t_{i}\right)
\end{gathered}
$$

Note: $\mathrm{AUC}_{\mathrm{G}}=$ area under the curve with regard to the ground, $A \cup C_{1}=$ area under the curve with regard to the increase, $m=$ repeated measure (i.e. at T1, T2, T3), $n=$ total amount of measurements; $\mathrm{t}=$ time distance

Fig. 2 Formulas for calculating the areas under the curve. Note: $\mathrm{AUC}_{\mathrm{G}}=$ area under the curve with regard to the ground, $\mathrm{AUC}_{\mathrm{I}}=$ area under the curve with regard to the increase, $\mathrm{m}=$ repeated measure (i.e. at $\mathrm{T} 1, \mathrm{~T} 2$, $\mathrm{T} 3$ ), $\mathrm{n}=$ total amount of measurements; $\mathrm{t}=$ time distance
TICS-SSCS can be predicted by the size of the weight bias internalization. The effects of the TSST over three measurement points were analyzed by the two-factorial ANOVA for repeated measures with between-factor group (WBI-category) and within-factor time. In addition, a twofactorial ANCOVA for repeated measures was performed, including the following covariates: depression, age, gender and group size. If the Mauchly-Test indicated that sphericity was violated, the Greenhouse-Geisser correction generated by SPSS was used.

\section{Results}

\section{Descriptive analysis}

Table 2 gives an overview of the sociodemographic characteristics of the sample under investigation, including information on health and internalization. The scores reflecting weight bias internalization ranged between 0.2 and 5.2. With respect to gender, for female participants, the mean score was slightly higher (WBI: Ø2.9) compared with male participants (WBI: Ø2.3). This is similar to a study by Hilbert et al. (2014), in which female participant scored 2.90 on average, whereas male participants score 2.44 on average. Overall, the majority $(60.8 \%)$ of participants in this sample can be categorized with medium internalization (Table 2). The Screening Scale measuring Chronic Stress (SSCS) revealed high levels of stress in this sample with a mean score of 32 compared with standard scores of this scale (Schulz \& Schlotz, 1999).

\section{Cortisol measurements}

Table 3 and Fig. 3 summarize results of the salivary cortisol from three different time-points as well as including the areas under the curve for the overall sample as well as for each group of internalization separately (low, medium, and high). Results show that individuals with lower levels of internalized stigmatization have higher cortisol levels at the beginning of the TSST-G (T1); however, this difference was not significant (NS) $(\mathrm{F}(2,76)=1.08 ; p=\mathrm{NS})$. Significant differences can be found directly after the test phase at $\mathrm{T} 2(\mathrm{~F}(2,76)=4.78 ; p=$ $0.011)$, as well as during the recovery phase at $\mathrm{T} 3(\mathrm{~F}(2,76)=$ 4.20; $p=0.019)$. Participants with low internalization have higher cortisol responses than participants with moderate or high internalization.

Additionally, participants were categorized into responders and nonresponders using the cutoff $1.5 \mathrm{nmol} / \mathrm{L}$. According to this cutoff, $54.4 \%$ of the participants in this sample $(n=43)$ can be categorized as nonresponders, whereas $45.6 \%(n=36)$ can be categorized as responders. The mean weight bias internalization score for 
Table 3. Summary of Cortisol measurements and indices with regard to weight bias internalization category

\begin{tabular}{llll}
\hline & $\begin{array}{l}\text { AUC_G } \\
\text { mean (SD) }\end{array}$ & $\begin{array}{l}\text { AUC_I } \\
\text { mean (SD) }\end{array}$ & $\begin{array}{l}\text { SSCS } \\
\text { mean (SD) }\end{array}$ \\
\hline $\mathrm{WBI}_{\text {low }}$ & 423.7 (SD: 189.1) & 195.7 (SD: 181.3) & 25.1 (SD: 7.8) \\
$\mathrm{WBI}_{\text {medium }}$ & 272.1 (SD: 237.7) & 99.03 (SD: 175.2) & 32.8 (SD: 9.3$)$ \\
$\mathrm{WBI}_{\text {high }}$ & $203.1(\mathrm{SD}: 168.6)$ & $19.5(\mathrm{SD}: 126.6)$ & 35.3 (SD: 12.3$)$ \\
Significance* & $\mathrm{F}(2,76)=4.16 ; \mathrm{p}=0.019$ & $\mathrm{~F}(2,76)=4.26 ; \mathrm{p}=0.018$ & $\mathrm{~F}(2,76)=4.53 ; \mathrm{p}=0.014$ \\
\hline
\end{tabular}

WBI Weight bias internalization, AUC Area under curve, SD Standard deviation, SSCS Screening scale chronic stress, *Significance among WBIScategories using one-way ANOVA calculations, n.s. = not significant

nonresponders was 3.01 , and the mean score for responders was 2.29 , confirming the assumption that low or nonresponders have greater weight bias internalization compared with individuals categorized as normal responders.

\section{Two-factorial ANOVA/ANCOVA for repeated measurements}

Results of the two-factorial ANOVA for repeated measurements revealed a significant but small effect of time over the three measurement points $\mathrm{T} 1, \mathrm{~T} 2$, and $\mathrm{T} 3(\mathrm{~F}(1.463,111.157)=$ 13.397, $\left.p=0.000, \eta^{2}=0.150\right)$ and a significant, but small interaction effect time $\mathrm{x}$ group $(\mathrm{F}(2.925,111.157)=2.918, p=$ $\left.0.039, \eta^{2}=0.071\right)$. However, results of the two-factorial ANCOVA for repeated measurements, including the aforementioned covariates age, gender, group size, and depression reveal different findings. In this model the effect of time $\left(\mathrm{F}(1.442,103.816)=0.313, p=0.660, \eta^{2}=0.004\right)$, as well as the interaction effect time $\mathrm{x}$ group $(\mathrm{F}(2.884,103.816)=$ $\left.1.634, p=0.188, \eta^{2}=0.043\right)$ are not significant.

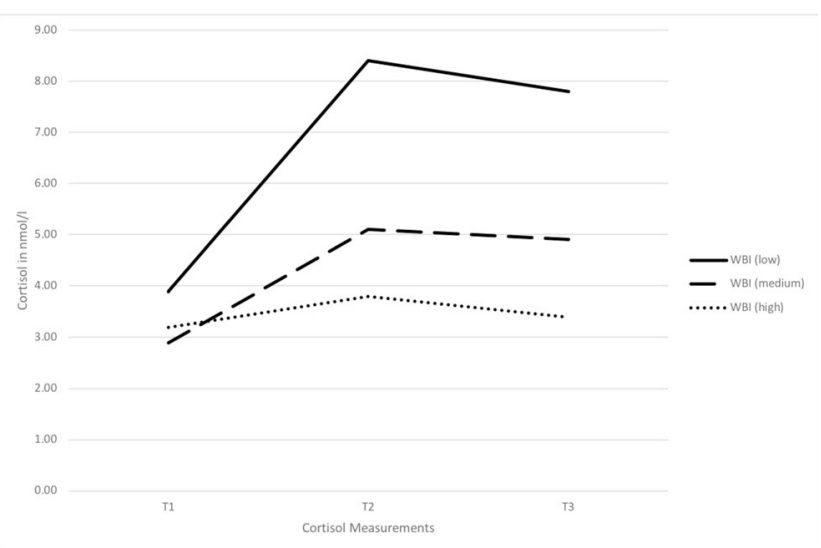

Note: Salivary cortisol was measured at three time points: T1, T2 and T3; all cortisol measurements in $\mathrm{nmol} / \mathrm{l} ; \mathrm{WBI}=$ weight bias internalization

Fig. 3 Cortisol responses after Trier Social Stress Test reveals differences among weight bias internalization categories. Note: Salivary cortisol was measured at three time points: T1, T2, and T3; all cortisol measurements in $\mathrm{nmol} / \mathrm{l} ; \mathrm{WBI}=$ weight bias internalization

\section{Experiences of chronic stress (TICS-SSCS)}

The prevalence of chronic stress in everyday life was assessed and evaluated with regard to the corresponding weight bias internalization category (low, medium, high; Table 3). Overall, the greater the weight bias internalization score, the greater the amount of chronic stress perceived by the participants in this study $\left(\mathrm{R}^{2}=0.1245, \mathrm{~F}(1,77)=10.95, p=0.001, \eta^{2}\right.$ $=0.124)$. Comparing the two measurements with regard to the area under the curve $\left(\mathrm{AUC}_{\mathrm{G}}\right.$ and $\left.\mathrm{AUC}_{\mathrm{I}}\right)$, individuals with low internalization have greater AUCs compared with individuals with medium or high internalization. Especially the group of participants with the highest WBIS scores show a rather blunted cortisol response, indicated by small $\mathrm{AUC}_{\mathrm{I}}$ scores. In other words, individuals with higher WBIS scores have a smaller cortisol secretion over time (smaller increase in cortisol levels from baseline to peak, and/or form peak to recovery phase) and show less endocrinological responses to psychosocial stress.

\section{Discussion}

The purpose of the current study was to investigate whether weight bias internalization is characterized by changes in cortisol reactivity. Even if previous research indicates that rising cortisol levels during psychosocial stressors are common among individuals with obesity in general, the role of internalization has hardly been investigated. Contrary to our hypothesis, results show that individuals with higher weight bias internalization scores do not significantly show higher basal cortisol compared with individuals with lower weight bias internalization. Due to the literature on weight stigma and cortisol, we expected that participants with greater internalization show greater cortisol levels at baseline (Himmelstein et al., 2015; Jackson et al., 2016). Our results rather demonstrate that experiencing weight stigma and internalizing weight bias represent two different constructs, aligning with the literature (Pearl, Puhl, \& Dovidio, 2015). In other words, experiencing weight stigma may have different physiological consequences on the human body than internalization. 
However, the results of this study reveal differences with regard to the stress response and an imbalance of the HPA stress axis. Individuals with higher internalized weight bias can be categorized as low or nonresponders after being exposed to a psychosocial stressor due to blunted cortisol responsivity. This aligns with a very recent study that has shown that participants who rate themselves as overweight or who were overweight according to their weight and height exhibit blunted cortisol responses in an experimental session that included weight stigmatization (McCleary-Gaddy et al., 2019).

The present study suggests that individuals with greater weight bias internalization have lower cortisol reactivity in response to an acute stressor; however, these significant results vanish after introducing covariates (gender, age, group size, and depression). Group size may have influenced the cortisol response, because the time differed between measurement at T1 and T2;. However, this would only affect the measurement straight after TSST at T2, because recovering phase was equal for every participant in this study. According to a study that investigated whether TSST with multiple participants (individual, groups of two or three participants) may influence psychopathological mechanisms found that it did not significantly affect cortisol response (Childs, Vicini, \& de Wit, 2006). Another reason could be that it may have an impact whether two or three participants are present during the TSST-G. Social-evaluative threat may increase with the group size or, on the other hand, may be smaller, when the number of peers being present is greater (Dawans et al., 2011). However, with regard to social threat, stress depends on the amount of elements of uncontrollability (i.e., audience, video camera, and another participants) rather than the element itself (Dickerson \& Kemeny, 2004).

An imbalance of the HPA stress axis may have negative impact on the distribution of adipose tissue, which is linked to comorbidities associated with obesity, such as cardiovascular disease and type 2 diabetes (Jackson et al., 2016; Fardet \& Fève, 2014). Apart from the literature already mentioned, hyporeactivity of the HPA axis has gained attention as an important cue for internalizing disorders, such as anxiety and depression (Bae, Stadelmann, Klein, Jaeger, Hiemisch, Kiess, 2015), which also are linked to obesity and weight discrimination (Myers \& Rosen, 1999).

Moreover, it has been shown (Henckens, Klumpers, Everaerd, Kooijman, van Wingen, Fernández, 2016) that even when higher basal cortisol levels correlate to stress resilience, increased stress-induced cortisol reactivity might be necessary to facilitate adequate recovery from stress, but only in individuals who react more sensitively to stressful events compared with others. In this context, a study investigating the link between cardiovascular reactivity and obesity in the Dutch Famine Birth Cohort (Phillips, Roseboom, Carroll, \& de Rooij, 2012) has shown that, indeed, decreased and not increased cortisol (and cardiac) stress reactivity is associated with obesity. They suggest that obesity could be associated with higher basal cortisol levels, but blunted cortisol reactivity in response to stressful events due to down-regulation of glucocorticoid-receptors or, alternatively, due to dysregulation of neural systems that induce motivated behavior (Hollon, Burgeno, \& Phillips, 2015; Lovallo, 2011).

Low cortisol levels might reflect an early termination of HPA axis, even if the individual under stress may still depend on elevated cortisol to activate resources successfully. Therefore, low cortisol levels mediate physiological disengagement, passive coping, and avoiding or ignoring important social cues. Cortisol reactivity is not found in $30 \%$ of participants in TSST situations (Allen et al., 2017). These so-called TSST nonresponders are hypothesized to show HPA dysregulation and failure of sufficient HPA stress response (Petrowski, Herold, Joraschky, Wittchen, \& Kirschbaum, 2010). Individuals show a rather low capacity to build up stress responses in a social context that requires it, lacking fidelity within the HPA axis. As a result, physiological functioning cannot be recalibrated to meet the demands of a changing environment of the individual, increasing the risk for stress-related diseases associated with adversely affecting this negative feedback system (Henckens et al., 2016). High levels of cortisol in response to psychosocial stressors might be good, because they allow us to be open to social information and interaction, being able to connect socially. Low or declining cortisol levels, however, indicate that the individual is socially or emotionally disengaged from its social environment (Shirtcliff, Vitacco, Graf, Gostisha, Merz, ZahnWaxler, 2009).

Another aspect that needs to be taken into consideration is called "biological silence." Based on their findings, Bourdarene et al. (2002) argue that individuals can be categorized into three groups according to their psychophysiological reactivity, which is based on the conclusion that emotional (i.e., anxiety) and biological reactions (i.e., plasma cortisol) can increase independently during stressful events. This suggests that people may respond with high anxiety even in the absence of cortisol levels, lacking a functional response to prepare dealing with a stressor (Boudarene, Legros, \& Timsit-Berthier, 2002). In other words, people who show emotional reactions to a stressor but simultaneously lack a biological response might be more psychologically vulnerable than individuals who respond on both axes accordingly.

Often, individuals with obesity and overweight experience weight discrimination and stigmatization in everyday life (Puhl \& Heuer, 2009). Previous research has shown that a history of negative experiences can impact how individuals evaluate and physiologically respond to social encounters (Iffland, Sansen, Catani, \& Neuner, 2014). A recent study has shown that a higher BMI in women was associated with blunted heart rate changes in response to negative social feedback if participants reported more emotional pain after weight- 
related teasing (Kube, Schrimpf, García-García, Villringer, Neumann, Horstmann, 2016). The authors argue that this may be an indicator of reduced salience of negative social information. Moreover, blunted cardiovascular responses are also linked to social exclusion (Newman, 2014). Social exclusion and isolation are very important in the stabilization of internalized weight bias and related negative consequences such as depression and impaired quality of life (Major, Dovidio, Link, Tomiyama, \& Hunger, 2017).

It has been shown with other types of stigmatization that experiences of stigmatizing situations can shape cortisol reactivity differently from what one might expect. A study by Hatzenbuehler et al. (2014) has shown that LGB young adults who grow up in highly stigmatizing surroundings substantiated blunted cortisol reactivity in response to the TSST compared with participants from low-stigmatizing social surroundings. Therefore, it might play a role for how long an individual with obesity has experienced weight stigma and whether he or she just started to internalize this weight bias or whether it has been present for a much longer period of time (i.e., since childhood). Additionally, the type of environment may play an important role in this context. Future studies should take this into account and investigate whether highstigmatizing environments and a long history of stigmatization and discrimination negatively affects cortisol responses during and after psychosocial stress.

\section{Limitations}

This study has limitations. First of all, before data was logarithmized, we observed some cortisol responders who did still show increasing levels of salivary cortisol despite being in the recovery phase. Additional saliva samples up to 1 hour after the TSST would provide better insight into the trajectory of cortisol reactivity. In addition, subjective stress experience induced by TSST-G was not included. Because our results appeared insignificant after introducing age, gender, depression, and group size during TSST-G, these variables should be treated as confounding variables. Especially the group-size variable may have influenced the results by leading to differences in time between measurements.

Second, information with regard to excluding factors were based on self-reports and the presence of eating disorders has only been assessed using one question. Weight and height also were based on self-reports. Several studies show a trend of under-reporting for weight and overreporting for height; however, this may still lead to accurate identification of overweight and obesity (Bowring, Peeters, Freak-Poli, Lim, Gouillou, Hellard, 2012). So far, there are no studies in Germany that document the magnitude of a possible bias (Maukonen, Männistö, \& Tolonen, 2018). Additionally, studies stress the importance of perceived weight and body image.
A very recent study has shown that both BMI and selfperceived weight moderate the relationship between weight bias and cortisol response and are associated with blunted cortisol responses (McCleary-Gaddy et al., 2019).

Future research may include a larger sample, being more able to differentiate alterations in cortisol response among different sociodemographic characteristics, such as age, gender, or weight history. As mentioned in the Discussion, socialization might play a role in shaping weight-bias internalization. Therefore, future studies should additionally ask for the social habitat of the individuals as well as the timeframe (or history) of weight discrimination.

\section{Conclusions}

The main findings of this study indicate that medium and high internalized stigmatization results in individuals being nonreactive to TSST. Therefore, the study contributes to the understanding of the psychological and endocrinological consequences of internalized weight bias and underlines the importance of interventions to reduce internalized stigmatization. Previous studies focused on weight discrimination as a chronic or acute stressor. However, it becomes clear that internalization seems to have effects on the individual that go beyond psychological consequences (i.e., lowered self-esteem, symptoms of depression) and negative health outcomes (i.e., weight cycling and weight gain). It therefore should be integrated in multidisciplinary weight management programs for individuals with overweight and obesity.

Acknowledgment This work was supported by the Federal Ministry of Education and Research (BMBF), Germany, FKZ: 01EO1501.

\section{References}

Abarca-Gómez, Leandra; Abdeen, Ziad A.; Hamid, Zargar Abdul; AbuRmeileh, Niveen M.; Acosta-Cazares, Benjamin; Acuin, Cecilia et al. (2017): Worldwide trends in body-mass index, underweight, overweight, and obesity from 1975 to 2016 . A pooled analysis of 2416 population-based measurement studies in 128.9 million children, adolescents, and adults. The Lancet 390 (10113), 2627-2642. DOI: 10.1016/S0140-6736(17)32129-3.

Allen, Andrew P.; Kennedy, Paul J.; Dockray, Samantha; Cryan, John F.; Dinan, Timothy G.; Clarke, Gerard (2017): The Trier Social Stress Test: Principles and practice. Neurobiology of stress 6, 113-126. DOI: https://doi.org/10.1016/j.ynstr.2016.11.001.

Bae, Yoon Ju; Gaudl, Alexander; Jaeger, Sonia; Stadelmann, Stephanie; Hiemisch, Andreas; Kiess, Wieland et al. (2016): Immunoassay or LC-MS/MS for the measurement of salivary cortisol in children? Clinical chemistry and laboratory medicine 54 (5), 811-822. DOI: https://doi.org/10.1515/cclm-2015-0412.

Bae, Yoon Ju; Stadelmann, Stephanie; Klein, Annette Maria; Jaeger, Sonia; Hiemisch, Andreas; Kiess, Wieland et al. (2015): The hyporeactivity of salivary cortisol at stress test (TSST-C) in children 
with internalizing or externalizing disorders is contrastively associated with $\alpha$-amylase. Journal of psychiatric research $71,78-88$. DOI: https://doi.org/10.1016/j.jpsychires.2015.09.013.

Boudarene, M.; Legros, J. J.; Timsit-Berthier, M. (2002): Etude de la réponse de stress: rôle de l'anxiété, du cortisol et du DHEAs. L'Encephale 28 (2), 139-146.

Bowring, Anna L.; Peeters, Anna; Freak-Poli, Rosanne; Lim, Megan Sc; Gouillou, Maelenn; Hellard, Margaret (2012): Measuring the accuracy of self-reported height and weight in a community-based sample of young people. BMC medical research methodology 12, 175. DOI: https://doi.org/10.1186/1471-2288-12-175.

Childs, Emma; Vicini, Lisa Marie; Wit, Harriet de (2006): Responses to the Trier Social Stress Test (TSST) in single versus grouped participants. Psychophysiology 43 (4), 366-371. DOI: https://doi.org/10. 1111/j.1469-8986.2006.00414.x.

Dawans, Bernadette von; Kirschbaum, Clemens; Heinrichs, Markus (2011): The Trier Social Stress Test for Groups (TSST-G): A new research tool for controlled simultaneous social stress exposure in a group format. Psychoneuroendocrinology 36 (4), 514-522. DOI: https://doi.org/10.1016/j.psyneuen.2010.08.004.

Dickerson, Sally S.; Kemeny, Margaret E. (2004): Acute stressors and cortisol responses: a theoretical integration and synthesis of laboratory research. Psychological bulletin 130 (3), 355-391. DOI: https:// doi.org/10.1037/0033-2909.130.3.355.

Durso, Laura E.; Latner, Janet D.; Hayashi, Kentaro (2012): Perceived discrimination is associated with binge eating in a community sample of non-overweight, overweight, and obese adults. Obesity facts 5 (6), 869-880. DOI: https://doi.org/10.1159/000345931.

Fardet, Laurence; Fève, Bruno (2014): Systemic glucocorticoid therapy: a review of its metabolic and cardiovascular adverse events // Systemic Glucocorticoid Therapy. A Review of its Metabolic and Cardiovascular Adverse Events. Drugs 74 (15), 1731-1745. DOI: https://doi.org/10.1007/s40265-014-0282-9.

Foss, Brynjar; Dyrstad, Sindre M. (2011): Stress in obesity: cause or consequence? Medical hypotheses 77 (1), 7-10. DOI: https://doi. org/10.1016/j.mehy.2011.03.011.

Gaudl, Alexander; Kratzsch, Juergen; Bae, Yoon Ju; Kiess, Wieland; Thiery, Joachim; Ceglarek, Uta (2016): Liquid chromatography quadrupole linear ion trap mass spectrometry for quantitative steroid hormone analysis in plasma, urine, saliva and hair. Journal of chromatography. A 1464, 64-71. DOI: https://doi.org/10.1016/j.chroma. 2016.07.087.

Hansen, Ase Marie; Garde, Anne Helene; Persson, Roger (2008): Sources of biological and methodological variation in salivary cortisol and their impact on measurement among healthy adults: a review. Scandinavian journal of clinical and laboratory investigation 68 (6), 448-458. DOI: https://doi.org/10.1080/ 00365510701819127.

Hatzenbuehler, Mark L.; McLaughlin, Katie A. (2014): Structural stigma and hypothalamic-pituitary-adrenocortical axis reactivity in lesbian, gay, and bisexual young adults. Annals of behavioral medicine: a publication of the Society of Behavioral Medicine 47 (1), 39-47. DOI: https://doi.org/10.1007/s12160-013-9556-9.

Henckens, Marloes J. A. G.; Klumpers, Floris; Everaerd, Daphne; Kooijman, Sabine C.; van Wingen, Guido A.; Fernández, Guillén (2016): Interindividual differences in stress sensitivity: basal and stress-induced cortisol levels differentially predict neural vigilance processing under stress. Social cognitive and affective neuroscience 11 (4), 663-673. DOI: https://doi.org/10.1093/scan/nsv149.

Herhaus, Benedict; Petrowski, Katja (2018): Cortisol Stress Reactivity to the Trier Social Stress Test in Obese Adults. Obesity facts 11 (6), 491-500. DOI: https://doi.org/10.1159/000493533.

Hilbert, Anja; Baldofski, Sabrina; Zenger, Markus; Löwe, Bernd; Kersting, Anette; Braehler, Elmar (2014): Weight bias internalization scale: psychometric properties and population norms. PloS one 9(1), e86303. DOI: 10.1371/journal.pone.0086303.
Himmelstein, Mary S.; Incollingo Belsky, Angela C.; Tomiyama, A. Janet (2015): The weight of stigma: cortisol reactivity to manipulated weight stigma. Obesity (Silver Spring, Md.) 23(2), 368-374. https://doi.org/10.1002/oby.20959.

Hollon, Nick G.; Burgeno, Lauren M.; Phillips, Paul E. M. (2015): Stress effects on the neural substrates of motivated behavior. Nature neuroscience 18 (10), 1405-1412. DOI: https://doi.org/10.1038/ nn.4114.

Iffland, Benjamin; Sansen, Lisa Margareta; Catani, Claudia; Neuner, Frank (2014): The trauma of peer abuse: effects of relational peer victimization and social anxiety disorder on physiological and affective reactions to social exclusion. Frontiers in psychiatry 5, 26. DOI: https://doi.org/10.3389/fpsyt.2014.00026.

Jackson, Sarah E.; Kirschbaum, Clemens; Steptoe, Andrew (2016): Perceived weight discrimination and chronic biochemical stress: A population-based study using cortisol in scalp hair. Obesity (Silver Spring, Md.) 24(12), 2515-2521. 10.1002/oby.21657.

Kirschbaum, C.; Pirke, K. M.; Hellhammer, D. H. (1993): The 'Trier Social Stress Test'-a tool for investigating psychobiological stress responses in a laboratory setting. Neuropsychobiology 28 (1-2), 7681. DOI: https://doi.org/10.1159/000119004.

Kirschbaum, C.; Prüssner, J. C.; Stone, A. A.; Federenko, I.; Gaab, J.; Lintz, D. et al. (1995): Persistent high cortisol responses to repeated psychological stress in a subpopulation of healthy men. Psychosomatic medicine 57 (5), 468-474.

Kube, Jana; Schrimpf, Anne; García-García, Isabel; Villringer, Arno; Neumann, Jane; Horstmann, Annette (2016): Differential heart rate responses to social and monetary reinforcement in women with obesity. Psychophysiology 53 (6), 868-879. DOI: https://doi.org/10. 1111/psyp.12624.

Lovallo, William R. (2011): Do low levels of stress reactivity signal poor states of health? Biological psychology 86 (2), 121-128. DOI: https://doi.org/10.1016/j.biopsycho.2010.01.006.

Löwe B, Spitzer RL, Zipfel S, Herzog W (2002): PHQ-D. Gesundheitsfragebogen für Patienten - Manual. Pfitzer.

Major, B., Dovidio, J.F., Link, B.G., Tomiyama, J., Hunger, J.M. (2017): The Negative and Bidirectional Effects of Weight Stigma on Health. Major B., Dovidio J.F., Link B.G. (Hg.): The Oxford Handbook of Stigma, Discrimination, and Health. 1. Aufl.: Oxford University Press.

Maukonen, Mirkka; Männistö, Satu; Tolonen, Hanna (2018): A comparison of measured versus self-reported anthropometrics for assessing obesity in adults: a literature review. Scandinavian journal of public health 46 (5), 565-579. DOI: https://doi.org/10.1177/ 1403494818761971.

McCleary-Gaddy, Asia T.; Miller, Carol T.; Grover, Kristie W.; Hodge, James J.; Major, Brenda (2019): Weight Stigma and HypothalamicPituitary-Adrenocortical Axis Reactivity in Individuals Who Are Overweight. Annals of behavioral medicine: a publication of the Society of Behavioral Medicine 53 (4), 392-398. DOI: https://doi. org/10.1093/abm/kay042.

McEwen, B. S. (1998): Stress, adaptation, and disease. Allostasis and allostatic load. Annals of the New York Academy of Sciences 840, 33-44.

McEwen, Bruce S. (2006): Protective and damaging effects of stress mediators: central role of the brain. Dialogues in clinical neuroscience 8 (4), 367-381.

Miller, Robert; Plessow, Franziska; Kirschbaum, Clemens; Stalder, Tobias (2013): Classification criteria for distinguishing cortisol responders from nonresponders to psychosocial stress: evaluation of salivary cortisol pulse detection in panel designs. Psychosomatic medicine 75 (9), 832-840. DOI: https://doi.org/10.1097/PSY. 0000000000000002 .

Myers, A.; Rosen, J. C. (1999): Obesity stigmatization and coping: relation to mental health symptoms, body image, and self-esteem. International journal of obesity and related metabolic disorders: 
journal of the International Association for the Study of Obesity 23 (3), 221-230.

Newman, Matthew L. (2014): Here we go agabullying history and cardiovascular responses to social exclusion. Physiology \& behavior 133, 76-80. DOI: https://doi.org/10.1016/j.physbeh.2014.05.014.

Nicolson, N. A. (2008): Measurement of Cortisol. In: Luecken, L.J., Gallo, L.C. (Hg.): Handbook of physiological research methods in health psychology. 1. Aufl.: SAGE Publications Inc., 37-74.

Papadimitriou, Anastasios; Priftis, Kostas N. (2009): Regulation of the hypothalamic-pituitary-adrenal axis. Neuroimmunomodulation 16 (5), 265-271. DOI: https://doi.org/10.1159/000216184.

Pearl, R. L.; Puhl, R. M. (2018): Weight bias internalization and health: a systematic review. Obesity reviews : an official journal of the International Association for the Study of Obesity 19 (8), 11411163. DOI: https://doi.org/10.1111/obr.12701.

Pearl, Rebecca L.; Puhl, Rebecca M.; Dovidio, John F. (2015): Differential effects of weight bias experiences and internalization on exercise among women with overweight and obesity. Journal of health psychology 20 (12), 1626-1632. DOI: https://doi.org/10. $1177 / 1359105313520338$.

Pearl, Rebecca L.; Wadden, Thomas A.; Hopkins, Christina M.; Shaw, Jena A.; Hayes, Matthew R.; Bakizada, Zayna M. et al. (2017): Association between weight bias internalization and metabolic syndrome among treatment-seeking individuals with obesity. Obesity (Silver Spring, Md.) 25(2), 317-322. DOI: 10.1002/oby.21716.

Peckett, Ashley J.; Wright, David C.; Riddell, Michael C. (2011): The effects of glucocorticoids on adipose tissue lipid metabolism. Metabolism: clinical and experimental 60 (11), 1500-1510. DOI: https://doi.org/10.1016/j.metabol.2011.06.012.

Petrowski, Katja; Herold, Ulf; Joraschky, Peter; Wittchen, Hans-Ulrich; Kirschbaum, Clemens (2010): A striking pattern of cortisol nonresponsiveness to psychosocial stress in patients with panic disorder with concurrent normal cortisol awakening responses. Psychoneuroendocrinology 35 (3), 414-421. DOI: https://doi.org/ 10.1016/j.psyneuen.2009.08.003.

Phillips, Anna C.; Roseboom, Tessa J.; Carroll, Douglas; de Rooij, Susanne R. (2012): Cardiovascular and cortisol reactions to acute psychological stress and adiposity: cross-sectional and prospective associations in the Dutch Famine Birth Cohort Study. Psychosomatic medicine 74 (7), 699-710. DOI: https://doi.org/10. 1097/PSY.0b013e31825e3b91.

Pruessner, Jens C.; Kirschbaum, Clemens; Meinlschmid, Gunther; Hellhammer, Dirk H. (2003): Two formulas for computation of the area under the curve represent measures of total hormone concentration versus time-dependent change. Psychoneuroendocrinology 28 (7), 916-931.

Puhl, Rebecca M.; Heuer, Chelsea A. (2009): The stigma of obesity: a review and update. Obesity (Silver Spring, Md.) 17(5), 941-964. DOI: $10.1038 /$ oby.2008.636.

Puhl, Rebecca M.; Himmelstein, Mary S.; Quinn, Diane M. (2018): Internalizing Weight Stigma: Prevalence and Sociodemographic Considerations in US Adults. Obesity (Silver Spring, Md.) 26 (1), 167-175. 10.1002/oby.22029.
Schommer, Nicole C.; Hellhammer, Dirk H.; Kirschbaum, Clemens (2003): Dissociation between reactivity of the hypothalamuspituitary-adrenal axis and the sympathetic-adrenal-medullary system to repeated psychosocial stress. Psychosomatic medicine 65 (3), 450-460.

Schulz, Peter; Schlotz, Wolff (1999): Trierer Inventar zur Erfassung von chronischem Streß (TICS). Skalenkonstruktion, teststatistische Überprüfung und Validierung der Skala Arbeitsüberlastung. Diagnostica 45 (1), 8-19. DOI: https://doi.org/10.1026//00121924.45.1.8.

Schvey, Natasha A.; Puhl, Rebecca M.; Brownell, Kelly D. (2014): The stress of stigma: exploring the effect of weight stigma on cortisol reactivity. Psychosomatic medicine 76 (2), 156-162. DOI: https:// doi.org/10.1097/psy.0000000000000031.

Shirtcliff, Elizabeth A.; Vitacco, Michael J.; Graf, Alexander R.; Gostisha, Andrew J.; Merz, Jenna L.; Zahn-Waxler, Carolyn (2009): Neurobiology of empathy and callousness: implications for the development of antisocial behavior. Behavioral sciences \& the law 27 (2), 137-171. DOI: https://doi.org/10.1002/bsl.862.

Spitzer, R. L.; Kroenke, K.; Williams, J. B. (1999): Validation and utility of a self-report version of PRIME-MD: the PHQ primary care study. Primary Care Evaluation of Mental Disorders. Patient Health Questionnaire. JAMA 282 (18), 1737-1744.

Tomiyama, A. Janet (2014): Weight stigma is stressful. A review of evidence for the Cyclic Obesity/Weight-Based Stigma model. Appetite 82, 8-15. DOI: https://doi.org/10.1016/j.appet.2014.06.108.

Tomiyama, A. Janet; Epel, Elissa S.; McClatchey, Trissa M.; Poelke, Gina; Kemeny, Margaret E.; McCoy, Shannon K.; Daubenmier, Jennifer (2014): Associations of weight stigma with cortisol and oxidative stress independent of adiposity. Health Psychology 33 (8), 862-867. DOI: https://doi.org/10.1037/hea0000107.

van der Valk, Eline S; Savas, Mesut; van Rossum, Elisabeth F C (2018): Stress and Obesity: Are There More Susceptible Individuals? Current obesity reports 7 (2), 193-203. DOI: https://doi.org/10. 1007/s13679-018-0306-y.

Woody, Alex; Hooker, Emily D.; Zoccola, Peggy M.; Dickerson, Sally S. (2018): Social-evaluative threat, cognitive load, and the cortisol and cardiovascular stress response. Psychoneuroendocrinology 97, 149155. DOI: https://doi.org/10.1016/j.psyneuen.2018.07.009.

Wu, Ya-Ke; Berry, Diane C. (2018): Impact of weight stigma on physiological and psychological health outcomes for overweight and obese adults: A systematic review. Journal of advanced nursing 74 (5), 1030-1042. DOI: https://doi.org/10.1111/jan.13511.

Wu, Ya-Ke; Berry, Diane C.; Schwartz, Todd A. (2019): Weight stigma and acculturation in relation to hair cortisol among Asian Americans with overweight and obesity: A cross-sectional study. Health psychology open 6(1), 2055102919829275. DOI: 10.1177/ 2055102919829275.

Publisher's note Springer Nature remains neutral with regard to jurisdictional claims in published maps and institutional affiliations. 